\title{
Self-monitoring of blood glucose: a promise still unfulfilled?
}

\author{
Simon R. Heller
}

Received: 3 January 2014 / Accepted: 7 January 2014 /Published online: 16 February 2014

(C) Springer-Verlag Berlin Heidelberg 2014

Keywords Glucose monitoring · Glycated haemoglobin · $\mathrm{HbA}_{1 \mathrm{c}} \cdot$ Randomised clinical trials $\cdot$ Self-monitoring blood glucose $\cdot$ Self-monitoring urinary glucose $\cdot$ Type 2 diabetes

\begin{abstract}
Abbreviations
RCT Randomised controlled trial

SMBG Self-monitoring of blood glucose
\end{abstract}

In the late 1970s, the introduction of self-monitoring of blood glucose (SMBG) promised a revolution in diabetes management for those taking insulin [1,2]. It paved the way for intensified insulin therapy in allowing a patient to measure their glucose level accurately and immediately and to confirm an episode of hypoglycaemia. Its availability led directly to the DCCT and other trials that established the benefit of keeping glucose close to normal. Yet, nearly 50 years later, the evidence to support the use of SMBG in type 1 diabetes is surprisingly limited. A fairly recent systematic review found no overall improvement in blood glucose control or significant reductions in hypoglycaemia in trials involving individuals with type 1 diabetes [3]. This may reflect the lack of interest in undertaking formal randomised controlled trials (RCTs) in an area where the advantages of such technology are thought to be obvious. Nevertheless, the failure of studies to demonstrate major benefits of established technologies in terms of blood glucose control or even hypoglycaemia in type 1 diabetes emphasises the methodological challenges in this area of research. It also suggests the likely difficulty in

\section{S. R. Heller $(\square)$}

Department of Human Metabolism, School of Medicine and

Biosciences, University of Sheffield, Beech Hill Road,

Sheffield S10 2RX, UK

e-mail: s.heller@sheffield.ac.uk establishing the value of SMBG in individuals with type 2 diabetes, who are at less risk of hypoglycaemia compared with those with type 1 diabetes [4] and where the link between a knowledge of current blood glucose is less obviously connected to changes in therapy.

To some extent this is confirmed by the publication in this issue of Diabetologia, of a recent trial of SMBG in individuals with type 2 diabetes using basic insulin therapy, either basal doses in combination with oral medication or twice daily premixed insulin preparations [5]. The trial was well designed. A 2 by 2 factorial design tested the effect of two interventions over 6 months in 300 individuals in different sites: (1) a once weekly SMBG profile vs no blood glucose monitoring and (2) a 3-monthly $\mathrm{HbA}_{1 \mathrm{c}}$ result available to the central laboratory but revealed to only half of the study centres.

The primary outcome, $\mathrm{HbA}_{1 \mathrm{c}}$ at 6 months, was no different between the arms, with a modest fall in $\mathrm{HbA}_{1 \mathrm{c}}$ from $7.3 \%$ to $7.0 \%$. The authors conclude that neither the use of SMBG nor the provision of $\mathrm{HbA}_{1 \mathrm{c}}$ results to centres was conducive to improving blood glucose control. However, they found that evidence of poor glycaemic control in either arm (most participants were measuring urinary glucose) generally led professionals to intensify therapy.

The authors also imply that because rates of hypoglycaemia were low in the trial, then providing blood glucose strips to detect hypoglycaemia was also not of great value. However, they stop short of recommending that SMBG should be withheld from this group of patients.

As in all trials there were limitations, some of which were acknowledged by the authors. Participants entered the study with reasonably tight blood glucose control, so there was limited scope for improvement. Patients who were frequently monitoring blood glucose were excluded from the trial, meaning that, in general, only those who did not find glucose monitoring useful were included. The method of SMBG amounted to an imposed 1-day-a-week profile, which may 
not have been the preferred way of monitoring for those participating. Finally, the number of dropouts was fairly high, amounting to just under $20 \%$ of those randomised.

What, then, are the clinical implications of this trial? By design, RCTs compare data from all participants randomised to different interventions and estimate the average effect of an intervention compared with a control group. Such an approach reduces bias, but although the average improvement in blood glucose control may be modest and includes individuals whose blood glucose control may improve by chance, it also includes participants whose blood glucose control improves markedly as a result of the intervention. Some individuals may find SMBG highly motivating and as a result alter their lifestyle which leads to improved glycaemic control (effects that are hidden in the overall result).

Qualitative research has confirmed that SMBG can help individuals with diabetes to confirm the relationship between symptoms and blood glucose, remind them of the potential seriousness of their condition, and motivate them to selfmanage their condition more effectively [6].

There is a concern that the data from the trial by Nauck et al [5] will be used to justify withholding or limiting testing strips from the minority who do find SMBG useful, particularly when health services are under financial pressure. On the other hand, the minimal impact of SMBG on $\mathrm{HbA}_{1 \mathrm{c}}$ in this trial and others $[7,8]$ also indicates that many of those with diabetes, even when treated with insulin, derive little benefit from SMBG in terms of either helping them to improve their blood glucose control or reducing hypoglycaemia. Nauck and colleagues hint (although do not state overtly) that such individuals might be advised not to monitor or could derive equal benefit from monitoring urine glucose (which is much cheaper). Indeed, SMBG may not only fail to help patients manage their diabetes more effectively, but can also generate adverse psychological effects [8].

There are a number of likely reasons as to why SMBG is rarely incorporated into successful diabetes self-management. Many patients make a note of the measurements without really understanding what they mean or how they should use the information. They are often frustrated when healthcare professionals take little interest in their diligence in recording blood glucose values [6]. Professionals frequently fail to explain how patients can use the results to identify glucose patterns that would allow them to self-adjust their insulin dose appropriately. However, perhaps the most important explanation for the limited effectiveness of SMBG is that monitoring is rarely integrated into a comprehensive and structured training programme in which these issues are expressly addressed.

Systematic reviews have confirmed the greater effectiveness of glucose self-monitoring when provided as part of an educational package, with overall improvements in $\mathrm{HbA}_{1 \mathrm{c}}$ of around $0.5 \%$. In the current trial, it appears that SBMG was not incorporated into a package of self-management, which may explain in part the limited improvement [9].

The value of integrating self-monitoring within a care package [10] has been also recognised in the UK by NICE, which in recent guidance, advises the need to assess at least annually the way in which self-monitoring is used, the use made of the results and the impact on quality of life [11].

The study of Nauck and colleagues emphasises that many years after the introduction of SMBG we still don't know how to use the potential of glucose monitoring even for individuals on insulin who stand to gain the most from knowing their blood glucose level. Yet, rather than using these negative results to justify limiting the availability of monitoring just to save money, we owe it to patients to respond more constructively.

It is the responsibility of diabetes specialists to ensure that people with diabetes are equipped with the knowledge to use self-monitoring more effectively. Front-line staff who interact with patients at a primary care level also need to know how self-monitoring fits within the overall care package. They should have sufficient expertise to discuss this with and support patients within the framework of a structured approach to the treatment of type 2 diabetes. This should include developing an individual approach to monitoring, encouraging those who find monitoring valuable in managing their condition, but stopping it in those whose blood glucose control is not improved or who experience deteriorating psychological outcomes. For these patients, SMBG might be used to identify hypoglycaemia in those at risk, with metabolic control monitored in other ways, perhaps urine testing, for whoever this is acceptable, together with regular measurement of $\mathrm{HbA}_{1 \mathrm{c}}$. The fact that $\mathrm{HbA}_{1 \mathrm{c}}$ provided no extra benefit in the participants with generally well-controlled glucose in the current trial is, in my view, not a reason to discard this measure of glycaemic control. As with SMBG, $\mathrm{HbA}_{1 \mathrm{c}}$ measurements were not integrated into a structured approach to care and, unlike urine testing, they can alert both patient and professional to an increased risk of hypoglycaemia.

More research is needed to establish both how SMBG is best integrated into self-management and whether the approach and self-management behaviours of those who use monitoring most effectively can be developed in others.

Finally, although it seems obvious that $\mathrm{HbA}_{1 \mathrm{c}}$ testing should be available for people with type 2 diabetes, we also need to understand how professionals and patients can make better use of these measurements.

Duality of interest The author declares that there is no duality of interest associated with this manuscript.

Contribution statement The author was the sole contributor to this paper. 


\section{References}

1. Sonksen PH, Judd SL, Lowy C (1978) Home monitoring of blood-glucose. Method for improving diabetic control. Lancet 1:729-732

2. Walford S, Gale EA, Allison SP, Tattersall RB (1978) Selfmonitoring of blood-glucose. Improvement of diabetic control. Lancet 1:732-735

3. Coster S, Gulliford MC, Seed PT, Powrie JK, Swaminathan R (2000) Monitoring blood glucose control in diabetes mellitus: a systematic review. Health Technol Assess 4:i-iv, 1-93

4. UK Hypoglycaemia Study Group (2007) Risk of hypoglycaemia in types 1 and 2 diabetes: effects of treatment modalities and their duration. Diabetologia 50:1140-1147

5. Nauck MA, Haastert B, Trautner C, Müller UA, Nauck MA, Heinemann L, for the clinical trials study group of the German Association for the Study of Diabetes (Deutsche DiabetesGesellschaft) (2014) A randomised, controlled trial of selfmonitoring of blood glucose in patients with type 2 diabetes receiving conventional insulin treatment. Diabetologia. doi:10.1007/ s00125-014-3168-1
6. Peel E, Parry O, Douglas M, Lawton J (2004) Blood glucose selfmonitoring in non-insulin-treated type 2 diabetes: a qualitative study of patients' perspectives. Br J Gen Pract 54:183-188

7. Farmer A, Wade A, Goyder E et al (2007) Impact of self monitoring of blood glucose in the management of patients with non-insulin treated diabetes: open parallel group randomised trial. BMJ 335:132

8. O'Kane MJ, Bunting B, Copeland M, Coates VE (2008) Efficacy of self monitoring of blood glucose in patients with newly diagnosed type 2 diabetes (ESMON study): randomised controlled trial. BMJ 336:1174-1177

9. An NHS Diabetes Working Group. (2009) Self monitoring of blood glucose in non-insulin-treated type 2 diabetes. Available from http:// webarchive.nationalarchives.gov.uk/20130316063827/, http://www. diabetes.nhs.uk/our_publications/reports_and_guidance/diagnosis and_ongoing_care/\#, accessed 24 January 2014

10. Polonsky WH, Fisher L, Schikman $\mathrm{CH}$ et al (2011) Structured selfmonitoring of blood glucose significantly reduces A1C levels in poorly controlled, noninsulin-treated type 2 diabetes: results from the Structured Testing Program study. Diabetes Care 34:262-267

11. NICE (2009) NICE clinical guideline 87. Type 2 diabetes: the management of type 2 diabetes. National Institute of Clinical Excellence, London 